\title{
HPLC method for Pharmacokinetics of cis and trans isomer of cefprozil diastereomers in human plasma
}

\author{
Haojing Song, Guiyan Yuan, Chunmin Wei, Xiaoyan Liu, Rong Li, Benjie Wang, \\ Ruichen Guo* \\ Institute of Clinical Pharmacology, Qilu Hospital of Shandong University, Jinan 250012, P.R. of China
}

\begin{abstract}
Using an high-performance liquid chromatography (HPLC) system and UV detection, a simple and precise analytical procedure was developed to quantify levels of cis and trans isomer of cefprozil diastereomers in human plasma. Cefprozil was extracted from plasma samples into $10 \%$ perchloric acid. The HPLC system included an acetonitrile- $0.05 \mathrm{M}$ monopotassium phosphate water (adjust pH to 3.05 with glacial acetic acid) $(12: 88, \mathrm{v} / \mathrm{v})$, flow rate of $1 \mathrm{~mL} / \mathrm{min}$, Ultimate ${ }^{\mathrm{TM}}$ $\mathrm{XB}-\mathrm{C} 18$ column, $(5 \mu \mathrm{m}, 4.6 \times 150 \mathrm{~mm})$, and UV detection at $280 \mathrm{~nm}$. The regression of the spiked calibrator curves were linear from $0.02-10 \mu \mathrm{g} / \mathrm{mL}$ and $0.02-1 \mu \mathrm{g} / \mathrm{mL}\left(\mathrm{r}^{2}=0.9951,0.9950\right)$, respectively. The lower limits of quantification were 0.02 $\mu \mathrm{g} / \mathrm{mL}$, the inter- and intra-day precisions (RSD) were lower than $9.0 \%$, and the extraction recoveries were all more than 90\%. The cis and trans isomers of cefprozil were stable under a variety of storage and process conditions. To demonstrate utility, the pharmacokinetic parameters of cis and trans isomer of cefprozil were measured in twenty human after oral administration of 500mg cefprozil. The assay was sensitive, accurate and convenient, and can be used for the determination of cis and trans isomer of cefprozil in human plasma.
\end{abstract}

Key words: cefprozil; cis and trans isomer; pharmacokinetics; HPLC

\section{Introduction}

Cefprozil, is considered a second-Generation cephalosporin. It is an oral cephaloporin with a broad in vitro spectrum of resistant against both Gram-positive (GP) and Gram-negative bacteria (GN) by inhibiting bacterial peptidoglycan cell wall synthesis and penicillinase [1-3].

Cefprozilisa mixture of cis and trans isomers in an approximately 9:1 ratio. The major isomer has the 3-(1-propenyl) substituent in the cis configuration. The chemical stability and the pharmacological activity of resistant GP, streptococcus and staphylococcus activity of cis isomer is equal to trans isomer, but the pharmacological activity of resistant GN of cis isomer is 6-8 times as same as trans isomer [2].

The principal aim of this study is to develop and validate a method for quantifying cefprozil diastereomers in human plasma. Because both isomers exhibit antimicrobial activities, it is necessary to determine the pharmacokinetics of each isomer respectively. To date, there have been few published methods for the measurement of cefprozi levels in biological tissue [4,5].

Some studies had been developed for the determination and quantification of cefprozil diastereomers separately [4,5]. Although these assays suggested a highly specific and precise bioanalytical method, there were some limitations such as run times were longer than $15 \mathrm{~min}$, and the extract method was complex. Moreover, the low limit of quantification was established to be $100 \mathrm{ng} / \mathrm{mL}$ and $20 \mathrm{ng} / \mathrm{mL}$ for cis and trans isomers. However, the MICs of cefprozil against upper

* Corresponding author: Ruichen GUO, Institute of Clinical Pharmacology, Qilu Hospital of Shandong University, Jinan 250012, P.R. of China. 
respiratory infections were in the range of $0.016-4 \mathrm{mg} / \mathrm{L}$ which was important considerations for therapy [6], a suitable quantification method was needed to be developed to lower the limit of quantification and improve the sensitivity of cefprozil.

In this study, a simple, facility, and efficient HPLC method was developed, validated, and applied to the pharmacokinetics of cis and trans isomers of cefprozil in human plasma using fluconazol as an internal standard. The pharmacokinetic parameters would provide the basis for clinical rational drug use.

\section{Experimental}

\subsection{Chemicals and reagents}

The cis (LOT: H0E054) and trans (LOT: G0D341) isomers of cefprozil standards (Figure 1) were provided by USP ROCKVILLE. The internal standard, fluconazol (LOT: 100314-200503) were provided by the national institutes for food and drug control (Figure 1). Acetonitrile (HPLC grade, LOT: H31825) was purchased from J.T.BAKER (USA). Glacial acetic acid ( HPLC grade, LOT: 605041) was purchased from TEDIA company. Potassium dihydrogen phosphate (analytical grade, LOT: 20080313) was purchased from Laiyang fine chemical industry of Shandong Province. Perchloric acid (analytical grade, LOT: 20020814) was purchased from east chemical industry factory of Tianjin. Blank plasma was purchased from Qilu Hospital of Shandong University.

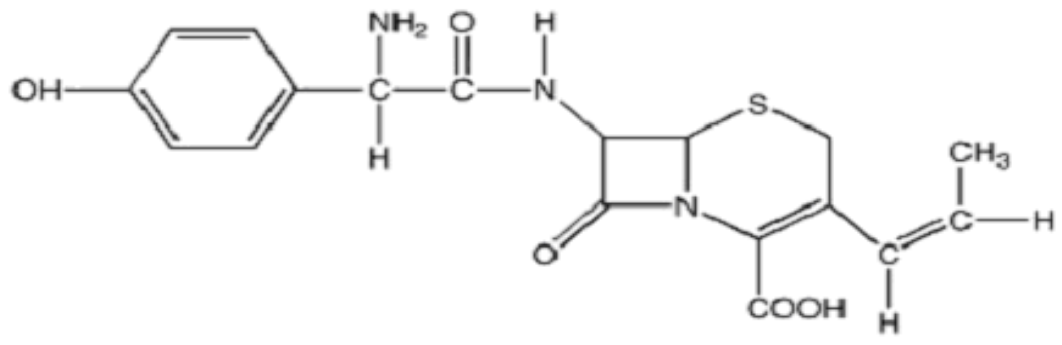

(A)

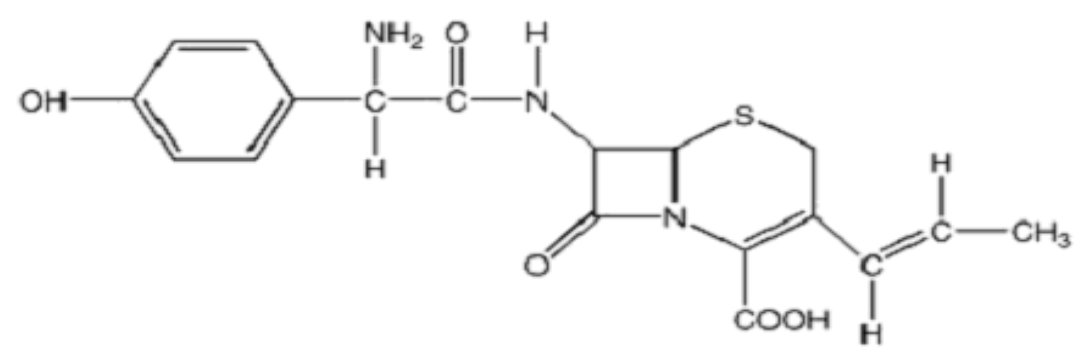

(B)

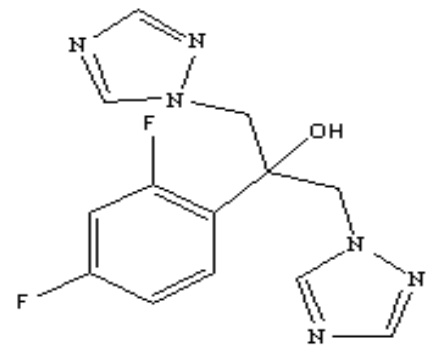

(C)

Figure 1 Chemical structures of (A) cis isomer of cefprozil, (B) trans isomer of cefprozil, and (C) fluconazol 


\subsection{Preparation of standard solutions}

Stock solutions of cis and trans isomers of cefprozil and IS were prepared in methanol at the concentration levels of 1.0 $\mathrm{mg} / \mathrm{mL}$. A series of standard solutions of cis and trans isomer of cefprozil at the concentration of $1,10,100 \mu \mathrm{g} / \mathrm{mL} \mathrm{were}$ obtained by step-wise dilution of the stock solution with mobile phase. Standard solution of IS at the concentration of 200 $\mu \mathrm{g} / \mathrm{mL}$ were obtained by dilute the stock solution with mobile phase. All the solutions were stored at $4{ }^{\circ} \mathrm{C}$, and were brought to room temperature before use.

The standard calibration samples of cefprozil were prepared by adding an appropriate volume of the standard solutions to $0.5 \mathrm{~mL}$ human plasma, giving the final concentrations of $0.02,0.05,0.1,0.5,1,5,10 \mu \mathrm{g} / \mathrm{mL}$ of $\mathrm{cis}$ isomers , and $0.02,0.05$, $0.1,0.2,0.5,1 \mu \mathrm{g} / \mathrm{mL}$ of trans isomers.

Quality control (QC) samples at the concentrations of $0.05,1,8 \mu \mathrm{g} / \mathrm{mL}$ of cis isomers and $0.05,0.2,0.8 \mu \mathrm{g} / \mathrm{mL}$ of trans isomers were prepared in the same way as the calibration standard and stored at $4{ }^{\circ} \mathrm{C}$.

\subsection{Chromatographic conditions}

The essebtial part of the HPLC system consisted of a Model Waters 515 pump, a Model Waters 717 autosampler, and a Model Waters 2487 Dual Wavelength Absorbance Detector.

The analtytic column was Ultimate TM XB-C18 column $(5 \mu \mathrm{m}, 4.6 \times 150 \mathrm{~mm})$. The separation was carried out with the mobile phase consisting of acetonitrile-0.05M monopotassium phosphate water (adjust to $\mathrm{pH} 3.05$ with glacial acetic acid) $(12: 88, \mathrm{v} / \mathrm{v})$ at a flow rate of $1.0 \mathrm{~mL} / \mathrm{min}$. The chromatograms were monitored at $280 \mathrm{~nm}$, and the column temperature was room temperature.

\subsection{Preparation of the samples}

To $0.5 \mathrm{~mL}$ plasma sample, $10 \mu \mathrm{L}$ IS and $100 \mu \mathrm{L} 10 \%$ perchloric acid were added. The solution was thoroughly vortex-mixed for $2 \mathrm{~min}$, and centrifugated at $10800 \mathrm{rpm}$ for $5 \mathrm{~min}$. $20 \mu \mathrm{L}$ supernatant were injected into the HPLC system for analysis.

\subsection{Preparation of biological samples}

The study was approved by the human ethics committee of Qilu Hospital of Shandong University.

Ten healthy male volunteers (aged 18-28 years) participated in the study. All subjects gave written informed consent. The studied men had no history of drug hypersensitivity, and they didn't take any medication in two weeks before entering and during the study. They were determined to be healthy by medical history, physical examination, and blood chemistry test.

After an overnight fast, the subjects received an oral dose of $500 \mathrm{mg}$ cefprozil with $200 \mathrm{~mL}$ water at 7 a.m. Blood samples were collected into heparinized tubes at $0,0.25,0.5,0.75,1.0,1.5,2.0,3.0,4.0,5.0,6.0,8.0,10.0$ and 12.0 $\mathrm{h}$ after dosing. Blood samples were centrifuged, and the plasma was separated. The plasma samples were kept frozen at $-20^{\circ} \mathrm{C}$ until assayed.

\section{Results}

\subsection{Method validation}

3.1.1. Specificity The specificity of the method was validated by comparing chromatograms of cis and trans isomers cefprozil and IS, blank plasma, blank plasma spiked with cis and trans isomers cefprozil and IS, and plasma from the healthy male volunteers (9-4) after oral $500 \mathrm{mg}$ cefprozil capsules. Retention times for cis and trans isomer of cefprozil diastereomers and fluconazol (internal standard) were 5.11, 7.38 and 10.36 min, respectively. Typical chromatograms were shown in Figure 2. 


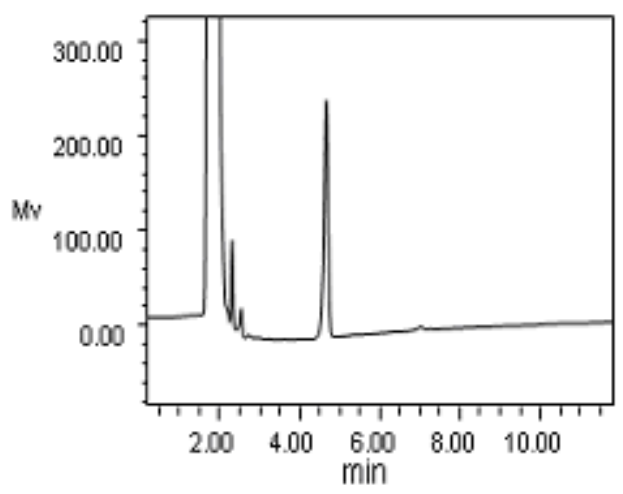

(a)

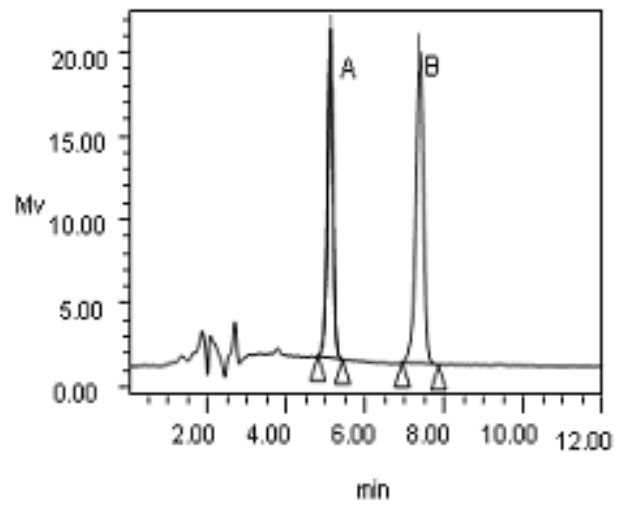

(b1)

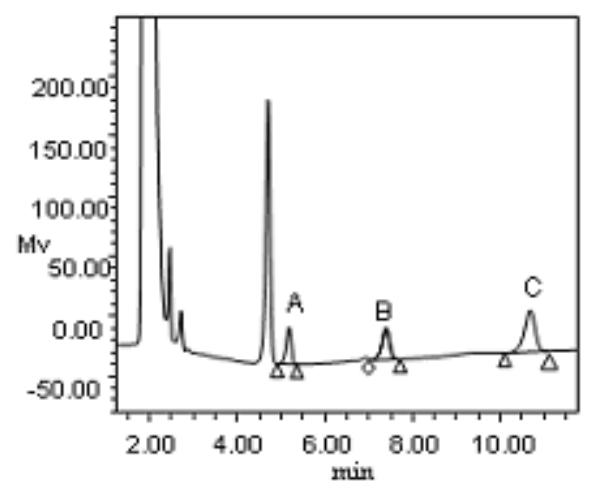

(c)

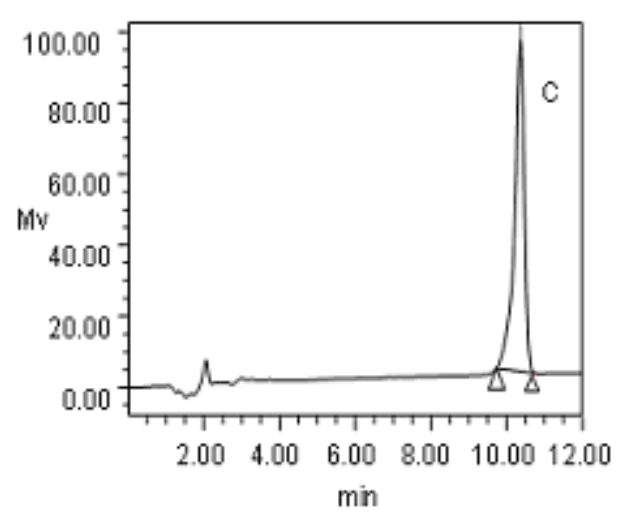

(b2)

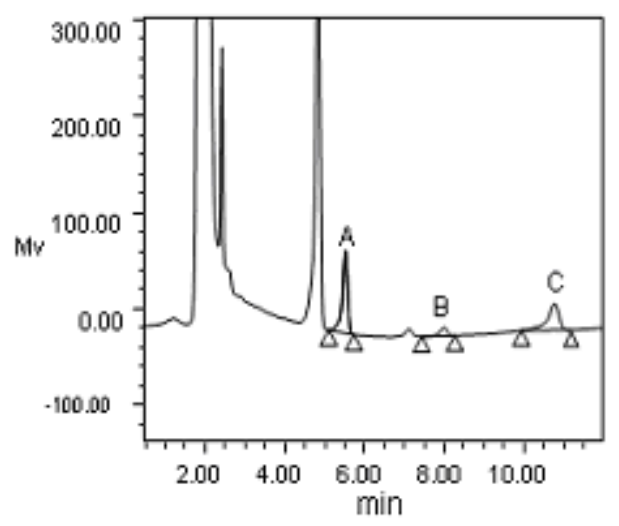

(d)

Figure 2 Chromatograms of blank plasma (a), cis and trans isomer of cefprozil (b1) and IS (b2), blank dog plasma spiked with cis and trans isomer of cefprozil and IS (c), plasma from a healthy male volunteer (9-4) at $1 \mathrm{~h}$ after oral cafprozil capsules with IS (d); A: cis isomer of cefprozil, B: trans isomer of cefprozil, C: IS

3.1.2. Low limit of quantification The lower limit of quantification (LLOQ) was defined as the lowest concentration of the calibration curve at which both precision and accuracy were less than $20 \%$. The current assay offered an LLOQ of 0.02 
$\mu \mathrm{g} / \mathrm{mL}$ in plasma of cis and trans isomers of cefprozil which were sufficient for the study of pharmacokinetics following a single oral administration of cefprozil.

3.1.3. Extraction recovery The extraction recoveries of cis and trans isomers of cefprozil were determined at three QC levels by comparing the peak areas of extracted plasma samples with those of extracted blank plasma spiked with corresponding concentrations. The extraction recovery of IS was also evaluated in the same way. The results showed the mean extraction recoveries of cis and trans isomers of cefprozil and IS were more than $90 \%$.

3.1.4. Linearity of calibration curve The calibration curves of cefprozil were produced by daily analysis of the spiked calibration samples at seven concentrations in the range of 0.02 to $10 \mu \mathrm{g} / \mathrm{mL}$ of cis isomer and 0.02 to $1 \mu \mathrm{g} / \mathrm{mL}$ of trans isomer. The linearity of calibration curves was assessed by linear regression with a weighting factor of the reciprocal of the concentration squared $\left(1 / \mathrm{x}^{2}\right)$. Therefore, the calibration curves were expressed by the equation of $\mathrm{Y}=1.71 \mathrm{X}+7.69 \times 10^{-3}$ $\left(\mathrm{R}^{2}=0.9951\right)$ and $\mathrm{Y}=2.12 \mathrm{X}-7.38 \times 10^{-3}\left(\mathrm{R}^{2}=0.9950\right)$, in which $\mathrm{y}$ represented the peak-area ratio and $\mathrm{x}$ represented the concentration ratio of cis or trans isomer of cefprozil to IS.

3.1.5. Accuracy and precision Accuracy, intra- and inter-day precisions were assessed with 5 replicates at concentrations of $0.05,1,8 \mu \mathrm{g} / \mathrm{mL}$ of cis isomer and $0.05,0.2,0.8 \mu \mathrm{g} / \mathrm{mL}$ of trans isomer on three constant days. The intraand inter-day precisions were expressed by relative standard deviation (RSD). The accuracy of the method was determined by calculating the percentage deviation observed in the analysis of QC samples. The results were shown in Table 1. It was suggested that the method was accurate and reproducible for the determination of cefprozil in human plasma.

Table 1 Inter- and intra-day precision and accuracy of cis and trans isomer of cefprozil $(\mathrm{n}=5)$

\begin{tabular}{|c|c|c|c|c|c|}
\hline & \multirow{2}{*}{$\begin{array}{l}\text { Concentration } \\
\qquad(\mu \mathrm{g} / \mathrm{mL})\end{array}$} & \multicolumn{2}{|c|}{ Precsion (\%) } & \multicolumn{2}{|c|}{ Acurracy (\%) } \\
\hline & & Intra-day & Inter-day & Intra-day & Inter-day \\
\hline \multirow{4}{*}{ Cis-cefprozil } & 0.02 & 5.90 & & 90.00 & \\
\hline & 0.05 & 2.71 & 5.51 & 107.63 & 103.62 \\
\hline & 1 & 5.46 & 5.21 & 109.06 & 104.42 \\
\hline & 8 & 4.31 & 3.01 & 93.58 & 94.70 \\
\hline \multirow{4}{*}{ Trans-cefprozil } & 0.02 & 9.48 & 3 & 95.00 & \\
\hline & 0.05 & 8.55 & 8.05 & 97.01 & 102.73 \\
\hline & 0.2 & 7.73 & 8.89 & 107.97 & 99.32 \\
\hline & 0.8 & 3.74 & 5.88 & 106.09 & 103.53 \\
\hline
\end{tabular}

3.1.6. Stability The stability of cis and trans isomer of cefprozil was investigated under a variety of storage and process conditions. The analytes were found to be stable after two freeze-thawing cycles $\left(-20{ }^{\circ} \mathrm{C}\right.$ to room teperature), and in the final plasma extract at room teperature for $8 \mathrm{~h}$. No signs of degradation were found under the freeze condition $\left(-20^{\circ} \mathrm{C}\right)$ for 30 days. The results were shown in Table 2. 
Table 2 stability of the samples

\begin{tabular}{|c|c|c|c|c|c|c|}
\hline & $\begin{array}{l}\text { Concentration } \\
\qquad(\mu \mathrm{g} / \mathrm{mL})\end{array}$ & Fresh sample & $\begin{array}{l}-20^{\circ} \mathrm{C} \\
\text { days }\end{array}$ & 30 & Two freeze-thawing & $\begin{array}{l}\text { Stored at room } \\
\text { temperature for } 8 \mathrm{~h}\end{array}$ \\
\hline \multirow{3}{*}{ Cis-cefprozil } & 0.05 & 106.23 & 95.95 & & 103.50 & 106.35 \\
\hline & 1 & 107.67 & 106.32 & & 102.90 & 105.90 \\
\hline & 8 & 98.43 & 104.64 & & 101.44 & 101.40 \\
\hline \multirow{3}{*}{ Trans-cefprozil } & 0.05 & 90.39 & 104.79 & & 87.80 & 101.25 \\
\hline & 0.2 & 107.29 & 97.40 & & 93.62 & 94.36 \\
\hline & 0.8 & 106.63 & 110.90 & & 100.55 & 98.54 \\
\hline
\end{tabular}

\subsection{Pharmacokinetic parameters}

The established HPLC method was successfully used to investigate the pharmacokinetics of the cis and trans isomer of cefprozil in human plasma, and Drug And Statistic Version 2.0 (DAS, 2.0, P.R. of China) was used to calculate the main pharmacokinetic parameters as shown in Table 3. The mean plasma concentration-time curves of cis and trans isomers of cefprozil were shown in Figure 3 and 4.

Tables 3 Main pharmacokinetic parameters of cis and trans isomer of cefprozil in human plasma (mean $\pm \mathrm{SD}, n=10)$

\begin{tabular}{lll}
\hline Parameter & Cis-cefprozi & Trans-cefprozi \\
\hline $\mathrm{V}(\mathrm{L} / \mathrm{kg})$ & $41.60 \pm 11.59$ & $41.66 \pm 11.73$ \\
$\mathrm{t}_{1 / 2}(\mathrm{~h})$ & $1.39 \pm 0.38$ & $1.11 \pm 0.38$ \\
$\mathrm{CL}(\mathrm{L} / \mathrm{h} / \mathrm{kg})$ & $21.02 \pm 4.17$ & $26.67 \pm 6.05$ \\
$\operatorname{Tmax}(\mathrm{h})$ & $1.83 \pm 0.52$ & $1.75 \pm 0.30$ \\
$\mathrm{AUC}_{0-\mathrm{t}}(\mathrm{mg} / \mathrm{L} * \mathrm{~h})$ & $22.13 \pm 4.57$ & $1.85 \pm 0.38$ \\
$\mathrm{AUC}_{0-\infty}(\mathrm{mg} / \mathrm{L} * \mathrm{~h})$ & $22.25 \pm 4.60$ & $1.97 \pm 0.45$ \\
$\mathrm{MRT}_{0-\mathrm{t}}(\mathrm{h})$ & $2.82 \pm 0.42$ & $2.41 \pm 0.31$ \\
$\mathrm{MRT}_{0-\infty}(\mathrm{h})$ & $2.89 \pm 0.46$ & $2.62 \pm 0.50$ \\
$\mathrm{Cmax}(\mathrm{mg} / \mathrm{L})$ & $7.20 \pm 1.24$ & $0.74 \pm 0.24$ \\
\hline
\end{tabular}

T1/2: terminal elimination half-life; $V$ : apparent volume of distribution; CL: clearance; AUC: area under the concentration-time curve; MRT: mean residence time; Cmax: peak concentration; Tmax: time of peak concentration. 


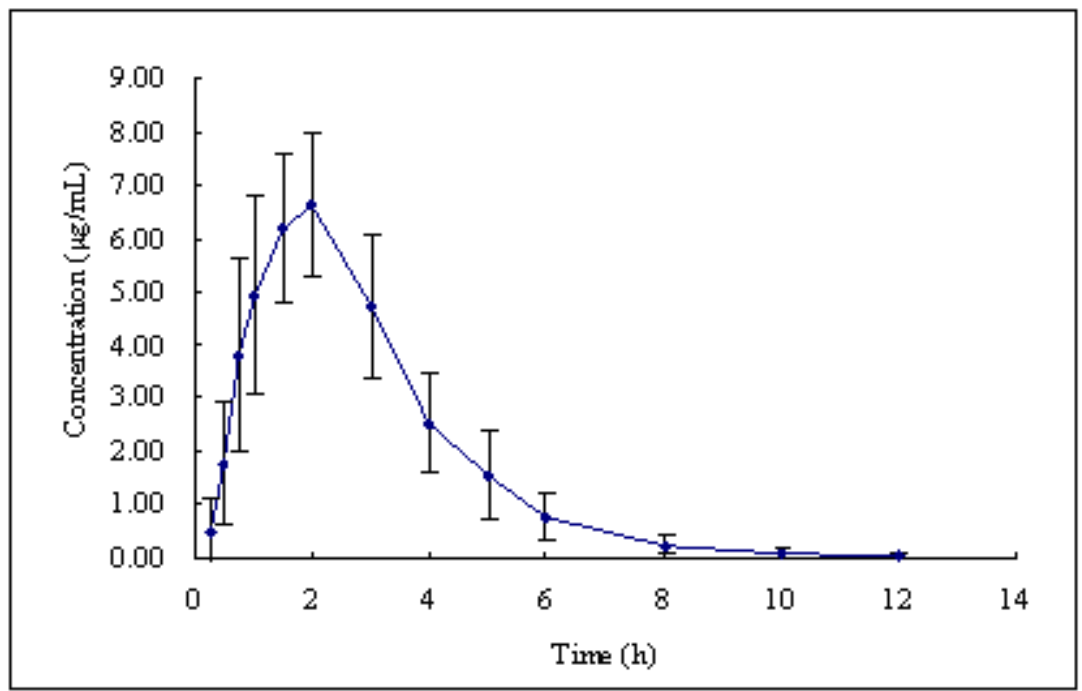

Figure 3 Mean plasma concentration-time curves of $c i s$-cefprozi (mean $\pm \mathrm{SD}, n=10$ )

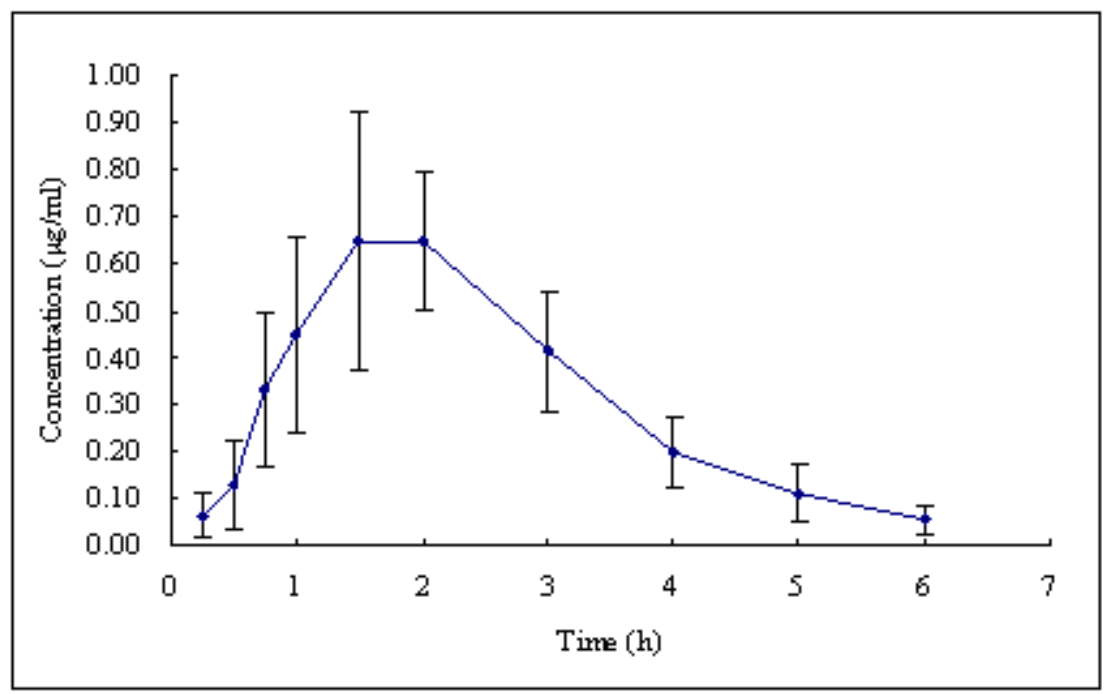

Figure 4 Mean plasma concentration-time curves of trans -cefprozi (mean $\pm \mathrm{SD}, n=10$ )

\section{Safety evaluation}

In the study, only one subject had nausea which continued for 1.5 hours but no affect to the trial. The medical results of 10 subjects after the study were normal.

\section{Discussion and conclusions}

A simple, facility and shortcut HPLC method was developed, validated, and applied to the pharmacokinetics of cis and trans-isomer of cefprozil diastereomers in human plasma after oral administration at dose of $500 \mathrm{mg}$.

Ultimate TM XB-C18 column $(5 \mu \mathrm{m}, 4.6 \times 150 \mathrm{~mm})$ was selected due to its excellent separation ability for cis and trans isomers, longer service life, and low expenses. Various mobile phase combinations of methanol and acetonitrile with different monopotassium phosphate and $\mathrm{PH}$ value were investigated to optimize sensitivity, speed and peak shape. Acetonitrile gave a better response than methanol, and $0.05 \mathrm{M}$ monopotassium phosphate water (adjust to $\mathrm{pH} 3.05$ with glacial acetic acid) improved peak shapes and the stability of cis and trans isomers of cefprozil.

In the study, liquid-liquid extraction and protein deposition were tested to extract cis and trans isomers of cefprozil. Liquid-liquid extraction was discarded because of impurities interference, low extraction recovery, and instability of cefprozil. 
Protein deposition method which was simple and facility, was used to dispose the plasma samples. Methanol, acetonitrile, and perchloric acid were tested as the precipitator. Finally, methanol and acetonitrile were discarded because of impurities interference, and $10 \%$ perchloric acid was chosen as the precipitator. The extraction procedure was simpler, more rapid, and higher recovery than the reported method [5].

Cefprozil consists of cis and trans isomers in an approximately 90:10 ratio. Since both isomers exhibit antimicrobial activities, the study analyzed the pharmacokinetic parameters of both isomers. The results showed that the pharmacokinetic parameters were no differences between cis and trans isomers except AUC and Cmax. The AUC values of cis isomer in plasma were about twelfth of that of trans isomer, and the Cmax values of cis isomer were about tenth of that of trans isomer. The result suggested that the metabolic processes of cis and trans isomer were approximately similar, and there were no transformation between cis and trans isomer in body.

Cefprozil was a second-generation cephalosporin frequently used in the treatment of community-acquired respiratorytract infections. The MICs of cefprozil against upper respiratory infections were in the range of $0.016-4 \mathrm{mg} / \mathrm{L}$ which was important considerations for therapy [6]. In this study, the plasma concentration of cis isomer susutained higher than $0.02 \mu \mathrm{g} / \mathrm{mL}$ for $12 \mathrm{~h}$, and of trans isomer susutained for $6 \mathrm{~h}$, which showed that the cefprozil had strong inhibition effect on sensitive bacteria. The results can be used to guide clinical medication for different upper respiratory infections.

The assay was sensitive, accurate and convenient, and can be used for the determination of cis and trans isomer of cefprozil in human plasma. The major advantages of the assay were lower LLOQ, simple sample preparation and a short run time. The metabolic processes of cis and trans isomer were approximately similar, and there were no transformation between the metabolism of cis and trans isomer. The results can be used to guide clinical medication for different upper respiratory infections.

\section{Reference}

1. Barriere SL. Review of in vitro activity,pharmacokinetic characteristics, safety,and clinical efficacy of cefprozil, a new oral cephalosporin. Ann Pharmacother, 27: 1082-1089 (1993).

2. Wiseman LR, Benfield P.Cefprozil. A review of its antibacterial activity, pharmacokinetic properties, and therapeutic potential. Drug, 45:295-317(1993).

3. Caroline M. Perry, Lesley J. Scott. Cefdinir,a review of its use in the management of mild-to-moderate bacterial infections. Drugs, 64:1433-1454 (2004).

4. Wen Chyi shyu, Umesh A.Shukla, Vinod R.Shah, et al. simultaneous high-performance liquid chromatographic analysis of cefprozil diasterepmers in a pharmacokinetic study. Pharmaceutical Research, 8:992-996(1991)

5. Tae-Hwan Park, Jin-Ki Kim, Jun-Pil Jee, et al. HPLC method for simultaneous determination of cefprozil diastereomers in human plasma. Journal of Pharmaceutical and Biomedical Analysis, 36:243-248(2004).

6. GAO Lei ,ZHANG Pu, LIU Yan, etal. Study on clinical pharmacokinetics and pharmacodynamics of cefprozil. Chin J Infect Chemother, 3:214-216 (2003). 